\title{
Towards an Image Blurring Detection Approach based on Color Filter Array
}

\author{
Mi Peng ${ }^{1}$, Luokai $\mathrm{Hu}^{1,2 *}$, Chao Liang ${ }^{2}$, Pingping Yin ${ }^{1}$ \\ ${ }^{1}$ Hubei Co-Innovation Center of Basic Education IT Services, Hubei University of Education, China. \\ ${ }^{2}$ Lenovo Mobile Communication Technology Co. Ltd., China. \\ *Corresponding Author e-mail:luokaihu@gmail.com
}

\begin{abstract}
Digital image tamper detection technology is a hot topic in recent decades which has widely applications in image processing. Study of key approach of digital image temper detection has important science and application value. A CFA interpolation based image blur tamper detection algorithm is proposed in this paper. First we reconstruct the tamper image by CFA interpolation artifacts. Then we filter the reconstructed image by Wiener filter and calculate the related degree between the tampering image and the image to be detected. Finally we classify the degree of correlation feature and locate the tampered area. Experimental result shows that the algorithm can effectively detect and locate tampering area of the image been operated by blur tampering and it has a good robustness. The algorithm can accurately locate the image of tampering falsification area and the detection accuracy is significantly better than existing algorithms and can be well positioned image tampering area.
\end{abstract}

Keywords- blur tamper detection; reconstructed; Wiener filter; the degree of correlation.

\section{INTRODUCTION}

In order to save costs, most digital cameras are produced by a single sensor to get full true color photos using the color filter array (CFA) and some interpolation algorithm. Image interpolation algorithms make pixels have some specific correlation and images have been tampered will most likely destroy or alter the correlation between those features are exploited to detect and locate the tampered image tampering.

A large number of algorithms on digital image forensics technology have been proposed in recent years. Bianchi exploited the JPEG image compression process and estimated the quantization step parameter model estimation methods [1]. Finally they used tamper area and not tampered area quantization table inconsistent locate tampered area. Peng et al [2] proved theoretically double JPEG image compression relationship between the first compression quantization tables and the second quantization tables. Then they used block artifacts locate tampered area and achieved a good results. Wei [3] used a simple way to detect the image re-sampling rate and the image rotation angle, if the image after JPEG compression, image resampling detection rate and the accuracy of the rotation angle of the image will be reduced. Chen et al used the statistical properties of the image after median filtering to detect whether the image is changed after tampering [4]. Wang Wei et al detected traces of fuzzy retouch images by treating the secondary side obfuscate and blur the image using the correlation achieved [5]. Fan et al used the image header file parameter tampering by the degree of change after the operation to detect whether the image is tampered after image forgery operation [6]. Kee et al used a simple way to extract tampering features inconsistency whether the image after tampering [7]. Wang et al. proposed a method for local histogram smoothing method based on the detection of the least significant bit of the image steganography has some effect, but the rate of detection accuracy is not very high [8]. Peng proposed a distribution and other inconsistencies to detect tampering image blur operation using the pixel direction [9, 10]. Different from above work, this paper proposed a detection method based on fuzzy CFA interpolation reconstruction.

Contrary to the above-mentioned disadvantages, this paper proposed a blurred trace detection method based on CFA interpolation reconstruction. We use the CFA interpolation detected image and its reconstructed Wiener filtering, reconstruct image and calculate the filtered image to be related to the degree of alteration, extract and classify the relevant features of the altered region of the positioning. The algorithm has very good effective for different types of fuzzy tampering.

\section{MONOCHROME SENSOR Digital CAMERA IMAGING PRINCIPLE}

Only one third digital pixel values are captured from the real scene. The remaining two thirds of the pixel values are estimated by interpolation algorithms combine color filter array are shown in Fig .1, the sensor can only collect accurate information for a single color channel and the pixel values of the rest of the missing data are combined with the color filter array interpolation algorithms estimated. The most common color filter array is the Bayer pattern. 


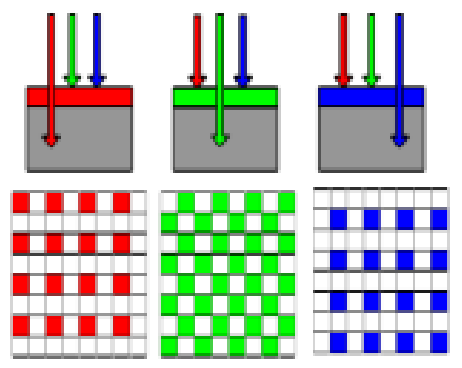

(a) Monochromatic device

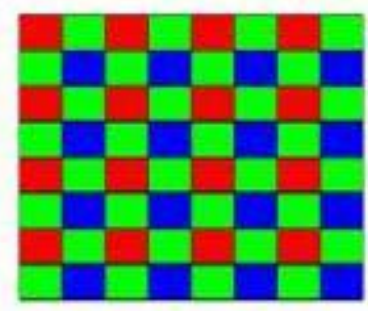

(b) Bayer pattern

Figure 1. Bayer model

The imaging process of monochrome sensor digital camera is as follows: First, the natural light entered digital camera through lens. Then the light is captured by monochrome sensor through various filters. However, monochrome sensor can only obtain the information from the intensity of color channels light. To obtain true color information, the light should pass through the color filter array first. The function of filter is passing the light with certain length, so as to achieve the required intake the purpose of the color. CFA is specified for each pixel on the sensor sampled in one of three primary colors, the pixel values of these interpolated samples, the formation of each individual color bit planes. There are many color interpolation algorithms based on smooth chroma interpolation algorithm, based on edge-aware interpolation algorithm and based on the weighted gradient directional interpolation algorithm. Different digital products use different interpolation technologies. After color interpolation and gamma correction, the image is written into camera memory with user-defined image formats (such as BMP, TIFF, JPEG, et. al.). The entire imaging process is completed.

\section{THE IMAGE CFA INTERPOLATION PROCESS}

Reconstruction for the Bayer matrix interpolation obtains all the values of $\mathrm{R}, \mathrm{G}, \mathrm{B}$ channel. $\mathrm{S}(\mathrm{x}, \mathrm{y})$ represents a Bayer matrix, R'(x,y), G'(x,y), B'(x,y) represents the original pixel value from the Bayer matrix which can be directly obtained $\mathrm{R}, \mathrm{G}, \mathrm{B}$ three-channel.

$$
\begin{gathered}
R^{\prime}(x, y)=\left\{\begin{array}{cc}
S(x, y), & S(x, y)=r_{x, y} \\
0, & \text { otherwise }
\end{array}\right. \\
G^{\prime}(x, y)=\left\{\begin{array}{cc}
S(x, y), & S(x, y)=g_{x, y} \\
0, & \text { otherwise }
\end{array}\right. \\
B^{\prime}(x, y)=\left\{\begin{array}{cc}
S(x, y), & S(x, y)=b_{x, y} \\
0, & \text { otherwise }
\end{array}\right.
\end{gathered}
$$

Reconstruction a full color image requires the use of the non-zero value interpolation of $R^{\prime}(x, y), G^{\prime}(x, y), B^{\prime}(x$, y) to reconstruct from the complete three color channels $\mathrm{r}(\mathrm{x}, \mathrm{y}), \mathrm{g}(\mathrm{x}, \mathrm{y}), \mathrm{b}(\mathrm{x}, \mathrm{y})$.

$$
\begin{aligned}
& r(x, y)=\sum_{u, v=-N}^{N} h_{r}(u, v) R^{\prime}(x-u, y-v) \\
& g(x, y)=\sum_{u, v=-N}^{N} h_{g}(u, v) G^{\prime}(x-u, y-v) \\
& b(x, y)=\sum_{u, v=-N}^{N} h_{b}(u, v) B^{\prime}(x-u, y-v)
\end{aligned}
$$

Where, $\operatorname{hr}(\cdot), \operatorname{hg}(\cdot), \operatorname{hb}(\cdot)$ represents the interpolation kernel for each color channel, the size is $(2 \mathrm{~N}+1) 2$. Different interpolation algorithms which interpolation different kernel functions, the use of a linear interpolation algorithm, the interpolation kernel function for green channel is hg, interpolation kernel function for the red channel is hr, blue channel interpolation kernel function is hb.

$$
\begin{aligned}
& h_{r}=\frac{1}{4}\left[\begin{array}{lll}
1 & 2 & 1 \\
2 & 4 & 2 \\
1 & 2 & 1
\end{array}\right] h_{g}=\frac{1}{4}\left[\begin{array}{lll}
0 & 1 & 0 \\
1 & 4 & 1 \\
0 & 1 & 0
\end{array}\right] h_{b}=\frac{1}{4}\left[\begin{array}{lll}
1 & 2 & 1 \\
2 & 4 & 2 \\
1 & 2 & 1
\end{array}\right] \\
& r(3,3)=r_{3,3} \quad, \quad g(3,3)=\frac{g_{2,3}+g_{3,2}+g_{3,4}+g_{4,3}}{4} \\
& b(3,3)=\frac{b_{2,2}+b_{2,4}+b_{4,2}+b_{4,4}}{4} \\
& r(3,4)=\frac{r_{3,3}+r_{3,5}}{2}, g(3,4)=g_{3,4}, b(3,4)=\frac{b_{2,4}+b_{4,4}}{2} \\
& r(4,4)=\frac{r_{3,3}+r_{3,5}+r_{5,3}+r_{5,5}}{4} \\
& g(4,4)=\frac{g_{3,4}+g_{4,3}+g_{4,5}+g_{5,4}}{4}, b(4,4)=b_{4,4} \\
& \begin{array}{|c|c|c|c|c|c|c|}
\hline r_{1,1} & g_{1,2} & r_{1,3} & g_{1,4} & r_{1,5} & g_{1,6} & \\
\hline g_{2,1} & b_{2,2} & g_{2,3} & b_{2,4} & g_{2,5} & b_{2,6} & \\
\hline r_{3,1} & g_{3,2} & r_{3,3} & g_{3,4} & r_{3,5} & g_{3,6} & \\
\hline g_{4,1} & b_{4,2} & g_{4,3} & b_{4,4} & g_{4,5} & b_{4,6} & \cdots \\
\hline r_{5,1} & g_{5,2} & r_{5,3} & g_{5,4} & r_{5,5} & g_{5,6} & \\
\hline g_{6,1} & b_{6,2} & g_{6,3} & b_{6,4} & g_{6,5} & b_{6,6} & \\
\hline & & & \vdots & & & \ddots \\
\hline
\end{array}
\end{aligned}
$$

Figure 2. Bayer matrix example

Fig .2 is a Bayer matrix $(3,3),(3,4)$ and $(4,4)$ pixels. Then the pixels of the interpolated correction shown in Fig .2 $(3,2)$ position of the interpolated $\mathrm{r}(3,2)$, and the original $g(3,2)$ respectively, the discrete wavelet transform, taking $\mathrm{r}(3,2)$ discrete wavelet transform low-frequency region and the high frequency region $\mathrm{g}(3,2)$ discrete wavelet transform wavelet inverse transform, thus corrected picture.

Shown in Fig .3, using the above algorithm in Fig .3 (a) are reconstructed to obtain Fig .3 (b), the algorithm can reproduce the original image, but not difficult to find at the edge of image detail, there will be vibration bell effect. And most of the existing image reconstruction algorithm is designed to improve the performance of the algorithm to reduce the ringing effect. 


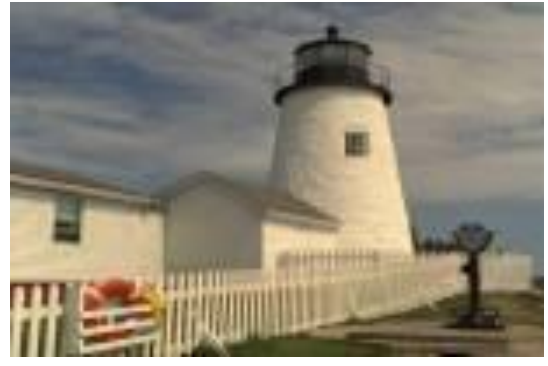

(a) original image

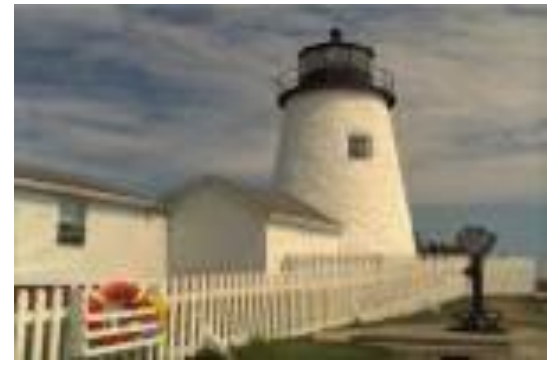

(b) reconstructed image

Figure 3. CFA Interpolation Reconstruction

The template is used to format your paper and style the text. All margins, column widths, line spaces, and text fonts are prescribed; please do not alter them. You may note peculiarities. For example, the head margin in this template measures proportionately more than is customary. This measurement and others are deliberate, using specifications that anticipate your paper as one part of the entire proceedings, and not as an independent document. Please do not revise any of the current designations. The reconstructed image obtained after the calculation of the Wiener filter to be tampered with the image correlation, the image is divided into blocks of $\mathrm{M}^{*} \mathrm{~N}$ overlapping size of the correlation calculation. $x_{i}$ is the reconstructed image to make the Wiener filter after the block of pixel values of the block, $y_{\mathrm{i}}$ is detected within the image sub-blocks of pixel values of the block.

$$
\begin{aligned}
& u_{1}=\frac{1}{M \times N} \sum_{i=1}^{M \times N} x_{i} \\
& u_{2}=\frac{1}{M \times N} \sum_{i=1}^{M \times N} y_{i} \\
& \sigma_{x}=\sqrt{\frac{1}{M \times N-1} \sum_{i=1}^{M \times N}\left(x_{i}-u_{1}\right)^{2}} \\
& \sigma_{y}=\sqrt{\frac{1}{M \times N-1} \sum_{i=1}^{M \times N}\left(y_{i}-u_{2}\right)^{2}} \\
& \sigma_{x y}=\frac{1}{M \times N-1} \sum_{i=1}^{M \times N}\left(x_{i}-u_{1}\right)\left(y_{i}-u_{2}\right) \\
& T=\frac{\sigma_{x y}}{\sigma_{x} \sigma_{y}}
\end{aligned}
$$

After getting the matrix T, we can categorize it into M1 and M2. M1 is the degree difference of T matrix which has not been tampered. M2 is degree difference of T matrix which has been tampered. Using EM method [1] to get its expectation and variance is as follows:

$$
P\left\{L(k, l) \mid M_{1}\right\}=N\left(u_{3}, \sigma_{1}\right)
$$

$$
P\left\{L(k, l) \mid M_{2}\right\}=N\left(u_{4}, \sigma_{2}\right)
$$

The feature matrix AL is as follows:

$$
A L=\frac{\left(L-u_{3}\right)^{2}}{2 \sigma_{1}^{2}}-\frac{\left(L-u_{4}\right)^{2}}{2 \sigma_{2}^{2}}
$$

Then matrix $\mathrm{AL}$ is filtered by the $3 \times 3$ median filter. The filtered result is processed with banalization, thereby obtaining the fuzzy tampered area.

\section{EXPERIMENTAL RESULTS AND ANALYSIS}

After the text edit has been completed, the paper is ready for the template. Duplicate the template file by using the Save As command, and use the naming convention prescribed by your conference for the name of your paper. In this newly created file, highlight all of the contents and import your prepared text file. You are now ready to style your paper. In order to verify the algorithm for image tamper detection of different types of fuzzy retouching effects, the paper size for a $512 \times 512$ image shown in Fig .4 (a) shows, using Photoshop image editing software for image processing face parts were used Gaussian blur lens blur, blur Motion blur blurred shapes and retouching process in which the corresponding parameters of each fuzzy

The pattern is shown in Table 1:

TABLE I. DIFFERENT FUZZY MODEL AND RELATED PARAMETERS

\begin{tabular}{|c|c|}
\hline Fuzzy pattern & Blur radius or distance \\
\hline Gaussian blur & 1 \\
\hline Lens blur & 5 \\
\hline Shape fuzzy & 5 \\
\hline Motion blur & 5 \\
\hline
\end{tabular}

Fig .3 (a) of the test image by fuzzy pattern different from the above four fuzzy treating the resulting image in Fig .3 retouch tampering (b) (e) below. The application referred to herein have tampered image detection algorithm based on fuzzy trace CFA interpolation reconstruction testing, test results of the experiment in Fig .3 $(\mathrm{g}) \sim(\mathrm{k})$ below. As can be seen from the detection result of the experiment, the grey zone and the pixel values after post-reconstruction image to be detected closer to the smaller effective reconstructed ringing generated, and in the detection result is a black area shows the study herein detection method has better detection results. The experimental results with the literature [5] contrast. 


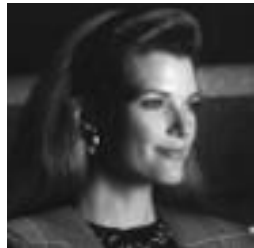

(a) Original

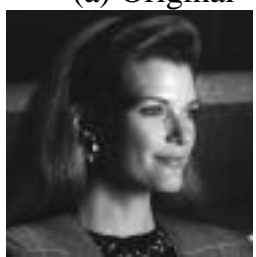

(d) the shape of fuzz
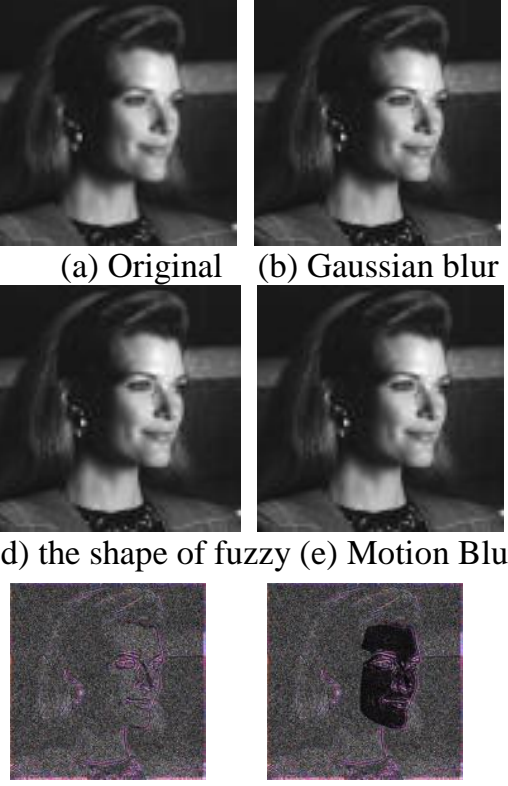

(b) Gaussian blur
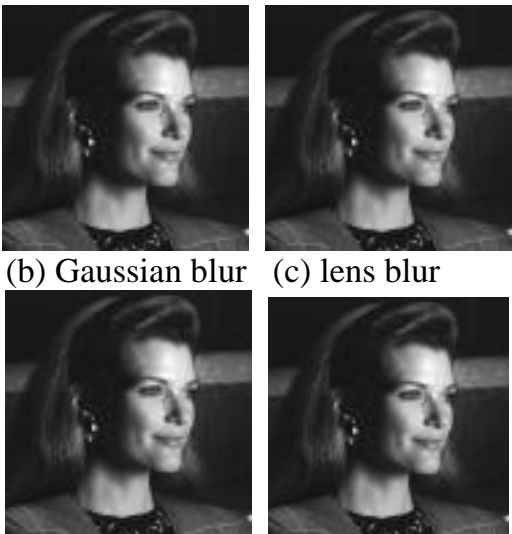

(c) lens blur

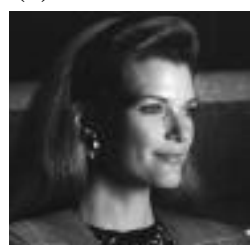

y (e) Motion Blur (f) Gaussian blur
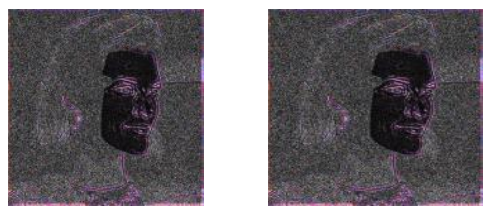

(g) Original test results (h) Gaussian Blur test results (i) lens blur test results
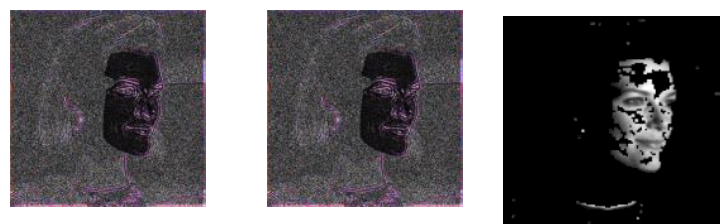

(j) the shape of fuzzy test results (k) Motion Blur test results (1) [5] test results

Figure 4. different types of test samples and fuzzy test results
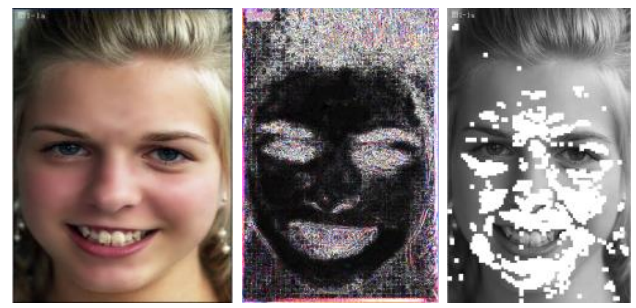

(a) tampering with the image (b) proposed algorithm test results (c) [5] test results

Figure 5. blurred image tamper detection results

Results Fig .4 and Fig .5 can be found in the literature [5] The algorithm can more accurately detect the tampered image tampering forgery area, but the result of detection, there are some false alarm region and the area of undetected. The proposed algorithm can accurately locate the image of tampering forged region, and the detection accuracy significantly better [5] is well positioned in the area of image tampering in the literature and the proposed algorithm. At the same time for different types of operations is fuzzy tampering with good results.

\section{CONCLUSION}

This paper discussed an image CFA interpolation algorithm based on fuzzy tamper detection. Firstly, the detected image is reconstructed using CFA interpolation effects. And then the detected image filtered by Wiener filter. Thirdly, we calculate its association degree between filtered reconstructed image and the original image. Finally, the feature of association degree is classified and tampered area will be located. Experimental results show that the algorithm for different types of fuzzy tampering and has a good effects.

\section{ACKNOWLEDGMENT}

This work was supported by a grant from the Hubei Provincial Department of Education scientific research programs for youth project (No. Q20133003) and the natural science foundation of Hubei Province (No. 2014CFB568).

\section{REFERENCES}

[1] Bianchi T, Piva A. "Image forgery localization via block-grained analysis of JPEG artifacts". IEEE Transaction on Information Forensics and Security, 2012, 7(3): 1003-1017.

[2] Yuanyuan Peng, Benyong Liu. "Accurate estimation of primary quantisation table with application to tampering". IET Electron Lett, 2013, 49(23): 1452-1454.

[3] Weimin Wei, Shuozhong Wang, Xinpeng Zhang. "Estimation of image rotation angle using interpolation-related spectral signatures with application to blind detection of image forgery". IEEE Trans on Information Forensics and Security, 2010, 5(3): 507-517.

[4] Chenglong Chen, Jiangqun Ni, Jixu Huang. "Blind Detection of Median Filtering in Digital Images: A Difference Domain Based Approach". IEEE Trans on Image Processing, 2013, 22(12): 46994710 .

[5] Wang Wei, Fang Yong. "secondary fuzzy correlation based single channel replacement Blind Image Separation". Applied Sciences, 2011, 29(2): 169-175

[6] Jiayuan Fan, Hong Cao, Alex C. "Estimating EXIF Parameters Based on Noise Features for Image Manipulation Detection". IEEE Transactions on Information Forensics and Security, 2013, 8(4): 608-618.

[7] Kee E, O'Brien J, FARID H. "Exposing Photo Manipulation with Inconsistent Shadows". ACM Transactions on Graphics, 2013, 32(4): 1-12.

[8] Wang Ran, Ping Xi-jian, Gong Zheng. "Two histogram-based local smoothness LSB matching analysis. Applied Sciences", 2012, 30(1): 96-104

[9] Peng Mi, Peng Yuanyuan. "Pixel image blur direction distribution inconsistent forensic method". Application of Electronic Technique 2014, 40(4): 63-66.

[10] G Cao, Y Zhao, R Ni. "Edge-based Blur Metric for Tamper Detection". Journal of Information Hiding and Multimedia, 2010, 1(1):20-27 